\title{
Quantum Correlations between Single Telecom Photons and a Multimode On-Demand Solid-State Quantum Memory
}

\author{
Alessandro Seri, ${ }^{1}$ Andreas Lenhard, ${ }^{1}$ Daniel Rieländer, ${ }^{1}$ Mustafa Gündoğan, ${ }^{1, \dagger}$ Patrick M. Ledingham, ${ }^{1, \$}$ \\ Margherita Mazzera, ${ }^{1, *}$ and Hugues de Riedmatten ${ }^{1,2}$ \\ ${ }^{1}$ ICFO-Institut de Ciences Fotoniques, The Barcelona Institute of Science and Technology, \\ Mediterranean Technology Park, 08860 Castelldefels (Barcelona), Spain \\ ${ }^{2}$ ICREA-Institució Catalana de Recerca i Estudis Avançats, 08015 Barcelona, Spain
} (Received 9 December 2016; revised manuscript received 30 March 2017; published 24 May 2017)

\begin{abstract}
Quantum correlations between long-lived quantum memories and telecom photons that can propagate with low loss in optical fibers are an essential resource for the realization of large-scale quantum information networks. Significant progress has been realized in this direction with atomic and solid-state systems. Here, we demonstrate quantum correlations between a telecom photon and a multimode ondemand solid state quantum memory. This is achieved by mapping a correlated single photon onto a spin collective excitation in a $\mathrm{Pr}^{3+}: \mathrm{Y}_{2} \mathrm{SiO}_{5}$ crystal for a controllable time. The stored single photons are generated by cavity-enhanced spontaneous parametric down-conversion and heralded by their partner photons at telecom wavelength. These results represent the first demonstration of a multimode on-demand solid state quantum memory for external quantum states of light. They provide an important resource for quantum repeaters and pave the way for the implementation of quantum information networks with distant solid state quantum nodes.
\end{abstract}

DOI: 10.1103/PhysRevX.7.021028

\section{INTRODUCTION}

Photonic quantum memories [1] are essential elements for quantum information networks [2], providing efficient and on-demand interfacing between single photons and stationary qubits, e.g., atomic gases [3-6], electronic spins in diamonds $[7,8]$, or phonons $[9,10]$. Besides featuring efficiencies and storage times comparable to or even surpassing those of atomic gases [11-14], solid state photonic memories based on rare-earth-doped crystals are becoming increasingly important as they offer prospects for scalability and integrability [15-18]. Most protocols for long-distance quantum communication require quantum memories connected to communication channels through optical fibers. One possible direction to achieve this goal is to use telecom quantum memories, e.g., based on erbium-doped solids

\footnotetext{
*margherita.mazzera@icfo.es

${ }^{\dagger}$ Present address: Cavendish Laboratory, University of Cambridge, J.J. Thomson Avenue, Cambridge CB3 OHE, United Kingdom.

${ }^{\star}$ Present address: Department of Physics, Clarendon Laboratory, University of Oxford, Oxford OX1 3PU, United Kingdom.

Published by the American Physical Society under the terms of the Creative Commons Attribution 4.0 International license. Further distribution of this work must maintain attribution to the author(s) and the published article's title, journal citation, and DOI.
}

Subject Areas: Photonics, Quantum Physics, Quantum Information
$[19,20]$ or optomechanical systems [10]. However, the most efficient and long-lived storage systems up to date are working at wavelengths far from the telecom window, leading to a large loss in optical fibers. Possible solutions to overcome this problem include quantum frequency conversion [3,21-25] or nondegenerate photon pair sources to establish entanglement between quantum memories and telecom photons [15,26-29]. The latter approach has been demonstrated using the atomic frequency comb scheme [30] in rare-earth-doped single crystals or waveguides $[15,27,28]$, but the storage of photonic entanglement has only been performed so far in the excited state for short and predetermined storage times.

Longer and programmable storage times can be obtained by transferring the optical atomic excitations to long-lived spin collective excitations [spin waves (SWs)] thanks to control laser pulses [30]. Recently, spin-wave storage of weak coherent states at the single-photon level [31,32], including qubit storage [31,33], has been demonstrated with rare-earth-doped crystals. The generation and storage of continuous variable entanglement between a multimode solid state quantum memory and a light field have also been reported recently [34]. In this experiment, lightmatter entanglement is created within the memory between spontaneously emitted light and spin waves, the matter part then being converted into a light field. This is a "readonly" quantum memory [1], with the generated light fields being resonant and, for this demonstration, outside the telecommunication band. This motivates the need for a 
"write-read" quantum memory that can store an externally prepared quantum optical state sharing a quantum correlation with a telecom band photon. To date, this has not been achieved with an on-demand spin-wave solid state quantum memory. Its successful realization with single photons requires an efficient quantum light source matching the spectral properties of the quantum memory $[35,36]$ and the quasisuppression of the noise generated by the strong control pulses. These tasks are challenging because of the small spectral separation between the hyperfine states of the optically active ions (a few $\mathrm{MHz}$ in our system).

Here, we demonstrate the spin-wave storage with on-demand retrieval of heralded single photons in a $\mathrm{Pr}^{3+}$ : $\mathrm{Y}_{2} \mathrm{SiO}_{5}$ crystal using the full atomic frequency comb (AFC) scheme. This is achieved by generating pairs of nondegenerate single photons where one photon is resonant with the optical transition of $\mathrm{Pr}^{3+}: \mathrm{Y}_{2} \mathrm{SiO}_{5}$ while the other photon is at telecom wavelength. The telecom photon is used to herald the presence of the other photon, which is stored as a SW in the crystal and retrieved on demand after a controllable time. We measure secondorder cross-correlation values between the heralding and the retrieved photons, which exceed the classical bound fixed by the Cauchy-Schwarz inequality for storage times longer than $30 \mu \mathrm{s}$, effectively demonstrating quantum correlations between telecom photons and single spin waves in a solid. Moreover, we demonstrate that our memory can store spin waves in multiple independent temporal modes.

\section{EXPERIMENTAL DETAILS}

Figure 1 depicts the experimental setup (a) and the relevant energy-level scheme of $\mathrm{Pr}^{3+}: \mathrm{Y}_{2} \mathrm{SiO}_{5}$ (b), where the chosen $\Lambda$ system is indicated by arrows. The atomic frequency comb is prepared, following the spectral hole-burning procedure described in Ref. [37], at the frequency of the $1 / 2_{\mathrm{g}}-3 / 2_{\mathrm{e}}$ transition, and the control pulses drive the coherence from the $3 / 2_{\mathrm{e}}$ to the empty $3 / 2_{\mathrm{g}}$ storage state and back. The narrow-band spectral filtering of the noise resulting from the control pulses is accomplished with a second $\mathrm{Pr}^{3+}: \mathrm{Y}_{2} \mathrm{SiO}_{5}$ crystal, where a narrow transparency window (around $5.5 \mathrm{MHz}$ ) is burned at the frequency of the AFC [31]. An example of AFC prepared in the memory crystal overlapped with the narrow spectral hole burned in the filter crystal is reported in Fig. 2(a).

Our cavity-enhanced SPDC source produces ultranarrow-band photon pairs, where one photon, the idler, is in the telecom E-band at $1436 \mathrm{~nm}$, and the other, the signal, is resonant with the $\mathrm{Pr}^{3+}$ optical transition at $606 \mathrm{~nm}$, specifically with the transition where the AFC is prepared [28,35]. The pump laser wavelength is $426.2 \mathrm{~nm}$, and the average power for the measurements presented in this paper is $3.3 \pm 0.5 \mathrm{~mW}$. The probability to obtain a single signal photon in front of the quantum memory conditioned on a detection in the idler SPD (i.e., the heralding efficiency) is $\eta_{H}=(20.9 \pm 0.5) \%$. We also switch off the SPDC pump after the detection of the idler photons for 30 to $40 \mu \mathrm{s}$ (depending on the experiment), thus interrupting the creation of photon pairs during the detection of the stored and

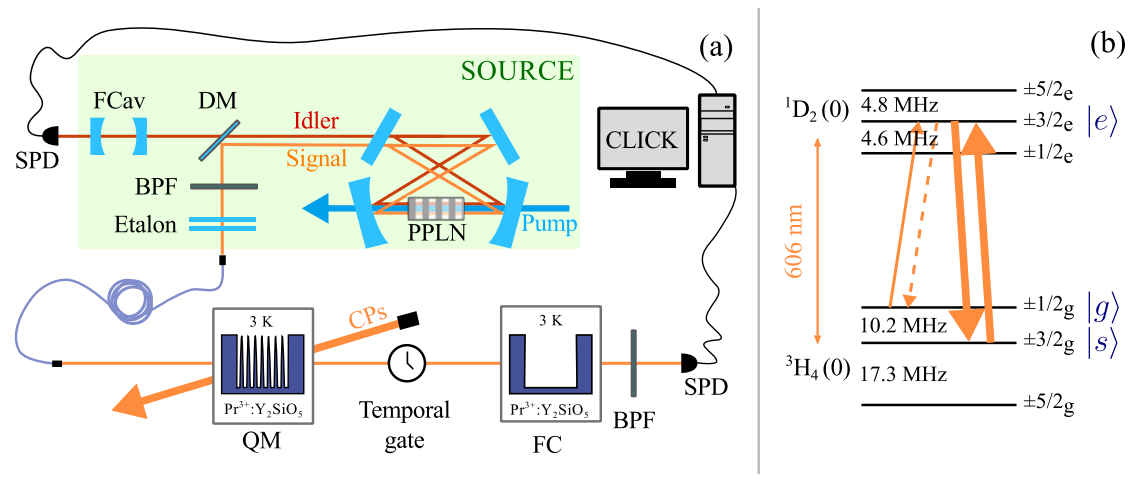

FIG. 1. (a) The experimental setup. Photon pairs are created by cavity-enhanced spontaneous parametric down-conversion (SPDC) in a periodically poled lithium niobate (PPLN) crystal. At the output of the cavity, they are separated by a dichroic mirror (DM). The idler photon is further filtered with a cavity (FCav) to select a single frequency mode, before being coupled in an optical fiber and detected with an InGaAs single-photon detector (SPD). The signal photon is spectrally filtered with a band-pass filter (BPF) and an etalon, before being coupled in a single-mode optical fiber and directed towards the quantum memory $(\mathrm{QM})$, where it is stored using the AFC scheme. Control pulses (CPs) are used to transfer optical excitations to spin waves and back; they are sent at an angle and counterpropagating with respect to the input photons. The retrieved single photon is spectrally filtered using a filter crystal (FC) before being detected by a silicon SPD. Temporal filtering is achieved with acousto-optic modulators, placed after the memory crystal, opened only when we expect the SW echo. This prevents additional hole burning in the filter crystal and the SPD to be blinded by eventual leakage of the control pulses. During the preparation of the memory crystal via optical pumping, the SPDs of both idler and signal arms are gated off. After each AFC preparation, the gates are opened, and we detect the arrival time of both photons of the pair during a measurement time of about $100 \mathrm{~ms}$, leading to a duty cycle of 0.14 . (b) Hyperfine splitting of the first sublevels of the ground ${ }^{3} \mathrm{H}_{4}$ and the excited ${ }^{1} \mathrm{D}_{2}$ manifold of $\mathrm{Pr}^{3+}$ in $\mathrm{Y}_{2} \mathrm{SiO}_{5}$. The arrows highlight the $\Lambda$ system chosen for the storage. 

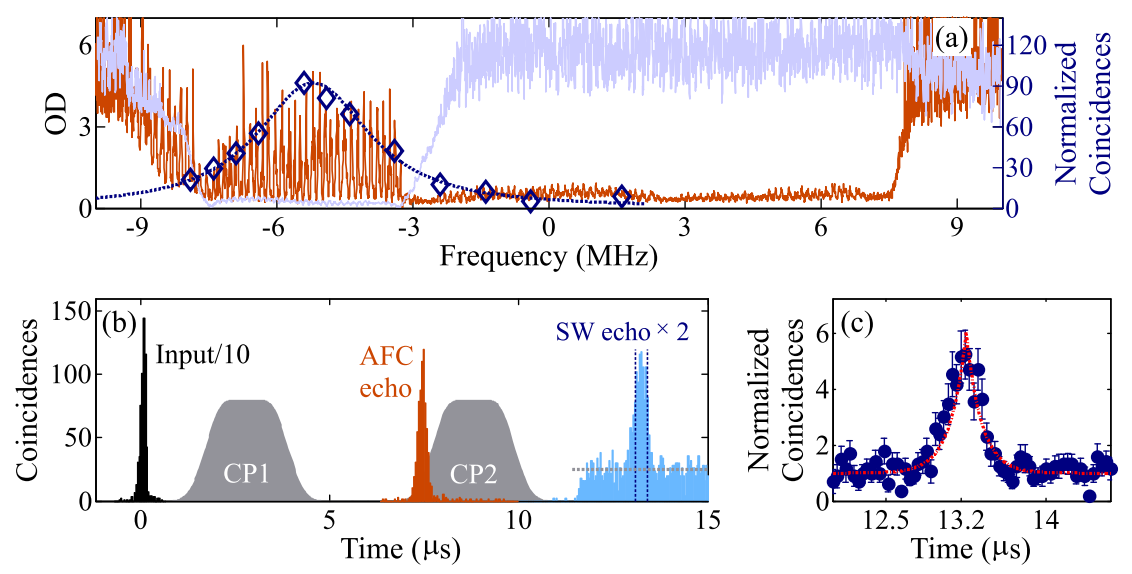

FIG. 2. (a) Example of an atomic frequency comb prepared for $\tau=7.3 \mu$ s (red trace). The light violet trace is the transparency window that we burn in the filter crystal. The diamonds are signal-idler coincidence rates taken after preparing a single 800-kHz broad transparency window in the memory crystal and moving its frequency along the input photons. The error is smaller than the data points. The dotted blue line is a simulation of a Lorentzian peak with FWHM $=2.8 \mathrm{MHz}$ convoluted with a $800-\mathrm{kHz}$-wide spectral hole. (b) Time histograms of the input photons (black trace), the AFC echo at $\tau=7.3 \mu \mathrm{s}$ (red trace), and the SW echo (blue trace) acquired over an integration time of $343 \mathrm{~min}$. We construct the coincidence histogram by taking the detection of the idler photons as a start and the detection of a signal photon after the memory as a stop. The coincidence count rate in the AFC echo is $4 / \mathrm{min}$ and in the SW echo $\approx 1 / \mathrm{min}$. The control pulses, detected with a reference photodetector, are displayed as plain pulses and are separated by $T_{s}=6 \mu \mathrm{s}$. They are modulated in amplitude and frequency with Gaussian and hyperbolic tangent waveforms, respectively, as described in Ref. [38]. The peak power is $21 \mathrm{~mW}$. The dashed vertical lines indicate the integration window for the signal $\left(\Delta T_{d}=320 \mathrm{~ns}\right)$, while the dashed horizontal line represents the noise floor. (c) Coincidence counts for the SW echo at $T=\tau+T_{s}=13.3 \mu$ s normalized by the average noise level, along with its fit to a double exponential function, to account for the Lorentzian spectral shape of the SPDC photons.

retrieved photons. Further details about the experimental setup and the preparation of the atomic frequency comb can be found in Ref. [38].

\section{HERALDED SINGLE-PHOTON SPECTRUM}

To access the spectrum of the heralded photons to be stored, we compare two measurements. First, we measure the temporal distribution of coincidences between the signal and idler photons of the source alone [36]. This correlation function has a width of $78 \mathrm{~ns}$ (FWHM), which is the correlation time of the photons. From this value, we calculate a biphoton bandwidth of $2.8 \mathrm{MHz}$. In a second experiment, we employ the $\mathrm{Pr}^{3+}: \mathrm{Y}_{2} \mathrm{SiO}_{5}$ memory crystal as a tunable frequency filter $[31,39,40]$. We prepare a $800-\mathrm{kHz}-$ wide spectral hole and record coincidence histograms when the central frequency is swept by about $10 \mathrm{MHz}$ around the frequency of the signal photons. In this experiment, the photons passing through the transparency window are directly steered to the APD for detection, bypassing the temporal and spectral filtering stages. The coincidence rate as a function of the hole position [blue diamonds overlapped with the AFC in Fig. 2(a)] gives the spectral distribution of the heralded single photons at $606 \mathrm{~nm}$. The result of this measurement agrees with the spectrum extrapolated from the signal-idler coincidence histogram measured immediately after the SPDC source [36]. This is confirmed by the good overlap between the diamonds and the blue dotted line, which represents the convolution of a Lorentzian curve of width $2.8 \mathrm{MHz}$ and the trace of the $800-\mathrm{kHz}$-wide spectral hole. We estimate the spectral overlap between the heralded single photons and the AFC to be about $70 \%$, which currently limits the AFC storage efficiency.

\section{SPIN-WAVE STORAGE OF HERALDED SINGLE PHOTONS}

We first measure the coincidence histogram when the signal photons are sent through a 18-MHz-wide transparency window prepared in the memory crystal [input trace at $0 \mu \mathrm{s}$ in Fig. 2(b)]. The correlation time between the signal and idler photons is $\tau_{c}=(89 \pm 4) \mathrm{ns}$, leading to a heralded photon line width of $(2.5 \pm 0.1) \mathrm{MHz}$ [36]. The correlation between the two photons is inferred by measuring the normalized second-order cross-correlation function $g_{s, i}^{(2)}=$ $P_{s, i} /\left(P_{s} \cdot P_{i}\right)$, where $P_{s, i}$ is the probability to detect a coincidence between idler and signal photons in a time window $\Delta T_{d}=320 \mathrm{~ns}$, while $P_{i}$ and $P_{s}$ are the unconditional probabilities to detect each photon. We find a $g_{s, i}^{(2)}$ value of $96 \pm 32$. Then, we prepare an AFC with periodicity $\Delta$ and store the single photon as a collective optical atomic excitation $\left|\psi_{e}\right\rangle=(1 / \sqrt{N}) \sum_{j=1}^{N} e^{i \mathbf{x}_{j} \cdot \mathbf{k}_{\text {in }}}\left|g_{1} \ldots e_{j} \ldots g_{N}\right\rangle$, where $\mathbf{k}_{\text {in }}$ is the wave vector of the incoming photon [see level scheme in Fig. 1(b)]. We obtain, for a preprogrammed storage time $\tau=(1 / \Delta)=7.3 \mu \mathrm{s}$, an efficiency $\eta_{\mathrm{AFC}}=(11.0 \pm 0.5) \%$ and $g_{\mathrm{AFC}, i}^{(2)}=130 \pm 31$ [see Fig. 2(b)]. This result represents an improvement in terms of $g_{\mathrm{AFC}, i}^{(2)}$ of more than 1 order of magnitude compared to the state of the art in the same system 
[28]. The $g^{(2)}$ value increases after the AFC storage due to the fact that the stored photons are transferred to a temporal mode free of noise [28]. After the retrieval, we measure $\tau_{c}=(147 \pm 7) \mathrm{ns}$, larger than the value measured before storage. We attribute this temporal stretching to spectral mismatch between the input photons before the memory $(\mathrm{FWHM}=2.8 \mathrm{MHz})$ and the atomic frequency comb (total width $4 \mathrm{MHz}$ ), as evidenced in Fig. 2(a).

We then perform spin-wave storage experiments by sending pairs of strong control pulses after the detection of each heralding photon. The first control pulse with wave vector $\mathbf{k}_{C}$ transfers the collective optical excitation $\left|\psi_{e}\right\rangle$ to a collective spin excitation (a spin wave), which can be written as $\left.\left|\psi_{\mathrm{sw}}\right\rangle=(1 / \sqrt{N}) \sum_{j=1}^{N} e^{i \mathbf{x}_{j} \cdot\left(\mathbf{k}_{\text {in }}-\mathbf{k}_{C}\right.}\right)\left|g_{1} \ldots s_{j} \ldots g_{N}\right\rangle$. For a storage time in the ground state of $T_{s}=6 \mu \mathrm{s}$ (thus, a total storage time $T=T_{s}+\tau=13.3 \mu \mathrm{s}$ ), we detect the retrieved photons, i.e., the spin-wave echo (swe), with an efficiency $\eta_{\mathrm{sw}}=(3.6 \pm 0.2) \%$. Note that $\eta_{\mathrm{sw}}$ is inferred with a coincidence window of $\Delta T_{d}=320 \mathrm{~ns}$, containing $80 \%$ of the signal, and it includes the loss in the filter crystal. Figure 2(c) shows a magnification of the SW echo mode, normalized by the average value of the noise measured outside the peak, leading directly to the signal-to-noise ratio (SNR) of the stored and retrieved photons. We observe a maximum SNR of around 5. This curve can also be used to $\operatorname{infer} g_{\mathrm{swe}, i}^{(2)}$. With our filtering strategy, we reach a noise floor of $(2.0 \pm 0.1) \times 10^{-3}$ photons per storage trial at the memory crystal (horizontal dashed line in the SW echo temporal mode). The correlation time of $\tau_{c}=(200 \pm 40) \mathrm{ns}$ exceeds the one after storage in the excited state. This further increase after the SW storage is attributed to the limited chirp of the control pulses (see Ref. [38]).

\section{QUANTUM CORRELATION BETWEEN SINGLE TELECOM PHOTONS AND SINGLE SPIN WAVES}

To investigate the nonclassical nature of the photon correlation after the SW storage, we measure $g_{\mathrm{swe}, i}^{(2)}\left(\Delta T_{d}\right)$ and compare it to the unconditional autocorrelation of the idler $\left[g_{i, i}^{(2)}\left(\Delta T_{d}\right)\right]$ and retrieved signal $\left[g_{\mathrm{swe}, \text { swe }}^{(2)}\left(\Delta T_{d}\right)\right]$ fields, respectively. To access these quantities, we correlate photon detections from different unconditional storage iterations [see Fig. 3(a)]. The time between two consecutive storage trials is $190 \mu \mathrm{s}$. In each trial (500 per comb preparation), we maintain the gate of the idler SPD open during $4.5 \mu$ s before sending the control pulses. We find $g_{\mathrm{swe}, i}^{(2)}=6.1 \pm 0.7$. We note that our $g_{\mathrm{swe}, i}^{(2)}$ is limited by the spin-wave read-out efficiency, which we estimate to be approximately

(b)

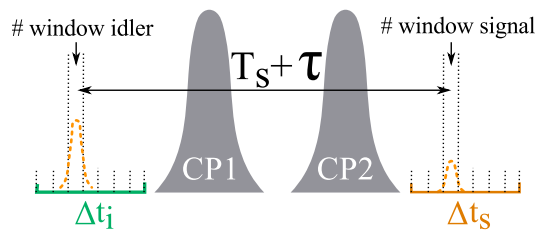

(c)

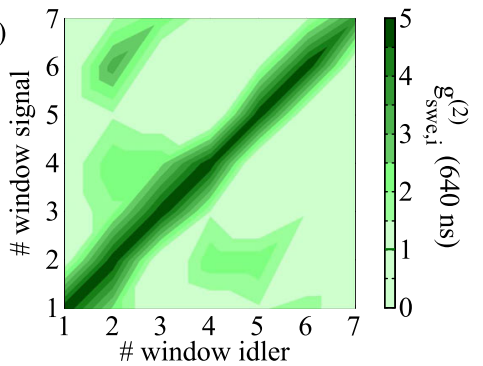

FIG. 3. (a) Unconditional cross-correlation between the idler photons and the retrieved signal photons. The $g_{\text {swe } i}^{(2)}\left(\Delta T_{d}=320 \mathrm{~ns}\right)$ value for this measurement is $6.1 \pm 0.7$. The classical bound given by the Cauchy-Schwarz inequality is reported as a horizontal line. The error bars are calculated considering Poissonian statistics. (b) Scheme representing the analysis of the cross-correlation measurement to evidence the multimodality. Both idler and retrieved signal integration windows are divided into smaller intervals (640 ns), and the correlation is calculated between intervals separated by different storage time. (c) Cross-correlation value between idler and retrieved signal photons detected in small temporal modes separated by different storage times. For this analysis, we also set $\Delta T_{d}=640 \mathrm{~ns}$ for better statistics. The $g_{\mathrm{swe}, i}^{(2)}\left(\Delta T_{d}=640 \mathrm{~ns}\right)$ exceeds the classical threshold for $\Delta T_{d}=640 \mathrm{~ns}$ (reported in the color bar) only for windows separated by the total storage time $T=13.3 \mu \mathrm{s}$. Violations outside these windows are not statistically significant because of a low number of counts. (d) Crosscorrelation value between the idler photons and the retrieved signal photons (full circles) and the coincidence counts in the SW echo (empty squares) as a function of the detection window for the idler photons. Both the signal detection window and the coincidence window remain constant at $4.5 \mu \mathrm{s}$ and $320 \mathrm{~ns}$, respectively. The classical threshold is also reported as a horizontal line. The integration time for this measurement is $38.5 \mathrm{~h}$. The error bars are calculated considering Poissonian statistics. 
$\eta_{R}=24 \%$, while the write efficiency is $\eta_{W}=31 \%$ (see Ref. [38] for a detailed discussion). With this unconditional sequence, the measured noise floor $\left[(1.3 \pm 0.1) \times 10^{-3}\right.$ photons per storage trial] is lower than with the conditional one (Sec. IV). We attribute this result to the fact that the number of control pulse pairs per comb is larger in the unconditional sequence. This probably contributes to further emptying of the spin storage state. The classical bound from the Cauchy-Schwarz inequality $g_{\mathrm{swe}, i}^{(2)}<\sqrt{g_{\mathrm{swe}, \mathrm{swe}}^{(2)} \cdot g_{i, i}^{(2)}}$ is indicated in Fig. 3(a) as a horizontal line. The measured unconditional autocorrelations are (also for $\Delta T_{d}=320 \mathrm{~ns}$ ) $g_{i, i}^{(2)}=1.32 \pm 0.04 \quad[36] \quad$ and $\quad g_{\text {swe,swe }}^{(2)}=1.0 \pm 0.4 \quad($ see Ref. [38]). The Cauchy-Schwarz parameter $R=\left(\left(g_{\mathrm{swe}, i}^{(2)}\right)^{2} /\right.$ $\left.g_{\mathrm{swe}, \mathrm{swe}}^{(2)} \cdot g_{i, i}^{(2)}\right)=28 \pm 12$ exceeds the classical limit of $R=1$ by more than 2 standard deviations. The confidence level for violating the Cauchy-Schwarz inequality, i.e., for observing a nonclassical correlation between the telecom heralding photon and the single spin wave stored in the crystal, is $98.8 \%$. If a larger coincidence window is considered, $\Delta T_{d}=1 \mu \mathrm{s}$, the $R$ value is reduced to $8.3 \pm 2.3$ because of a bigger contribution of noise in the $g_{\mathrm{swe}, i}^{(2)}$. On the other hand, because of a reduction of the statistical error, the confidence level for the demonstration of nonclassical correlation rises up to $99.92 \%$ (see Ref. [38]).

The main advantage of the full AFC protocol is the possibility to store multiple distinguishable temporal modes while maintaining their coherence and quantum correlation [30]. This ability is crucial for applications in quantum information protocols, e.g., to enable temporally multiplexed quantum repeater protocols with high communication speed [26] and storage of time-bin qubits robust against decoherence in optical fibers. To test this aspect, we perform experiments with detection gates much longer than the photon duration. We divide both the idler and the retrieved signal detection windows, $\Delta t_{i}$ and $\Delta t_{s}$, respectively, into smaller temporal modes of width $640 \mathrm{~ns}$, as sketched in Fig. 3(b). For this analysis, we also consider $\Delta T_{d}=640 \mathrm{~ns}$ in order to have better statistics. We then check that we have nonclassical correlations between modes separated by the total storage time $T=T_{s}+\tau=13.3 \mu$ s and classical correlations between modes separated by $T \neq 13.3 \mu \mathrm{s}$, as shown in Fig. 3(c). Contrary to other temporally multimode storage protocols $[11,34,41]$, in the full AFC protocol, the total storage time is maintained for the different temporal modes. To additionally demonstrate that the multimode capacity does not decrease the correlations, we compute $g_{\mathrm{swe}, i}^{(2)}\left(\Delta T_{d}=\right.$ $320 \mathrm{~ns})$ for idler detection windows $\Delta t_{i}$ varying from $320 \mathrm{~ns}$ to $4.5 \mu \mathrm{s}$, as shown in Fig. 3(d) (full circles), together with the coincidence counts measured in the center peak for each window size (empty squares). As expected, the latter increases linearly with $\Delta t_{i}$, but the $g_{\mathrm{swe}, i}^{(2)}$ value remains constant, within the error bar, and well above the classical bound over the whole range. Defining the number of temporal modes as $N_{m}=\Delta t_{i} / \Delta T_{d}$, we confirm nonclassical storage of a maximum of seven temporal modes with a $\Delta T_{d}=$ 640 ns that contains $94 \%$ of the coincidence peak. However, considering $\Delta T_{d}=320 \mathrm{~ns}$, which still contains $80 \%$ of the SW echo, a $4.5 \mu$ s-wide gate can accommodate up to 14 independent temporal modes.

Finally, to illustrate the ability to read out the stored spin wave on demand, we perform storage experiments at different SW storage times. For these measurements, we implement a semiconditional storage sequence in order to obtain good statistics with shorter integration time. We wait for heralding photons and, after each detection, we send 15 pairs of control pulses (each pair being separated from the neighboring one by $190 \mu \mathrm{s}$ ), which we exploit to correlate the idler and the retrieved signal (see Ref. [38]). The measured storage and retrieval efficiencies are reported in Fig. 4(a), together with a Gaussian fit, which accounts for the inhomogeneous broadening of the spin state. As a fitting parameter, we obtain the spin inhomogeneous line width $\gamma_{\text {inh }}=(20 \pm 3) \mathrm{kHz}$, in good agreement with that measured in different experiments on the same crystal $[18,31]$. This result further confirms that the photons are stored as spin waves. The second-order crosscorrelation function for increasing SW storage times $T_{s}$ is shown in Fig. 4(b). The red dashed curve is calculated by considering the Gaussian fit of the signal decay [solid curve in panel (a)], normalized by the source heralding efficiency $\eta_{H}$ and the average noise floor of $(1.9 \pm 0.2) \times 10^{-3}$ photons per trial (the average being over the different $T_{s}$ ). We measure
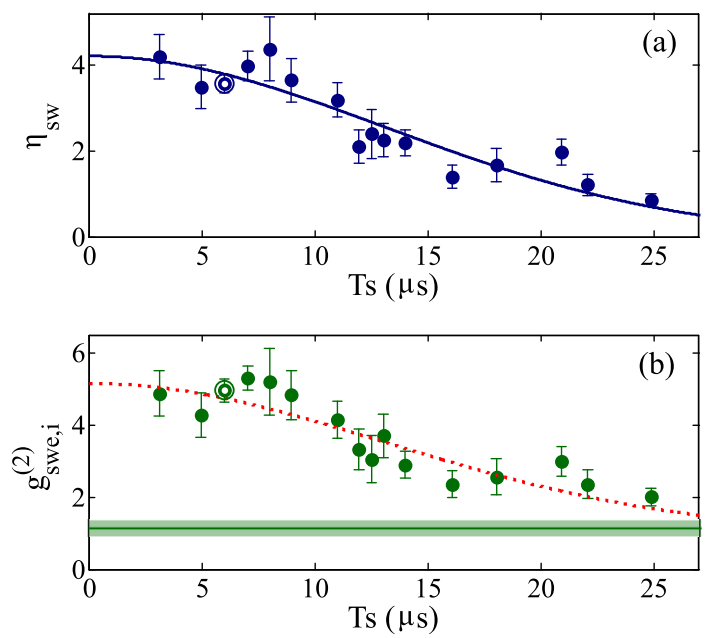

FIG. 4. (a) Spin-wave storage efficiency as a function of the storage time $T_{s}$. The solid line is a fit of the experimental data, taking into account a Gaussian inhomogeneous broadening of the spin state. The spin inhomogeneous broadening extracted from the fit is $\gamma_{\text {inh }}=(20 \pm 3) \mathrm{kHz}$. (b) The $g_{\text {swe }, i}^{(2)}(320 \mathrm{~ns})$ value as a function of the storage time. The classical threshold is reported as a horizontal line. In both panels, the error bars are calculated by considering Poissonian statistics, and the circled data points refer to the measurement reported in Fig. 2. 
nonclassical correlations between the idler and the retrieved signal photons up to a total storage time $T=\tau+T_{s}=$ $32.3 \mu$ s. Note that, while we measure the Cauchy-Schwarz parameter $R$ with unconditional measurements at $T=$ $13.3 \mu \mathrm{s}$, to assess the nonclassicality for longer storage times, we make the assumption that the retrieved signal autocorrelation does not increase for semiconditional measurements at different storage times. This is a conservative estimate since the $g_{\text {swe,swe }}^{(2)}$ value is mainly determined by the noise in the read-out, which we verify to be constant over the whole range of storage times investigated and not bunched (see Ref. [38]). Under this hypothesis, the Cauchy-Schwarz inequality is violated at $T=32.3 \mu$ s with a confidence of $94 \%$ for $\Delta T_{d}=320 \mathrm{~ns}$.

\section{DISCUSSION AND CONCLUSION}

The demonstrated quantum correlation between a telecom photon and a spin wave in a solid is an essential resource to generate entanglement between remote solid state quantum memories [26]. The measured value of $g_{\mathrm{swe}, i}^{(2)}$ after spin-wave storage is currently limited by the signal-tonoise ratio of the retrieved photon, which is, in turn, mostly limited by the low storage and retrieval efficiency. This value could be greatly improved by using higher optical depth [11] with higher-quality combs, or crystals in impedance matched cavities $[42,43]$. The storage time is currently limited by the spin inhomogeneous broadening and could be increased using spin-echo and dynamical decoupling techniques [32], with prospects for achieving values up to one minute [13] in our crystal, while even longer storage times (of order of hours) may be available in $\mathrm{Eu}^{3+}: \mathrm{Y}_{2} \mathrm{SiO}_{5}$ [14]. Finally, our experiment could be extended to the storage of entangled qubits, e.g., using time-bin encoding [31].

In conclusion, we have reported the first demonstration of quantum storage of heralded single photons in an on-demand solid state quantum memory. We have shown that the nonclassical correlations between the heralding and the stored photons are maintained after the retrieval, thus demonstrating nonclassical correlations between single telecom photons and single collective spin excitations in a solid. Finally, we showed that the full atomic frequency comb protocol employed allows one to store a single photon in multiple independent temporal modes. These results represent a fundamental step towards the implementation of quantum communication networks where solid state quantum memories are interfaced with the current fiber networks operating in the telecom window [26].

\section{ACKNOWLEDGMENTS}

We acknowledge financial support by the ERC Starting Grant QuLIMA, by the Spanish Ministry of Economy and Competitiveness (MINECO) and Fondo Europeo de Desarrollo Regional (FEDER) (FIS2015-69535-R), by
MINECO Severo Ochoa through Grant No. SEV-20150522 and through the Ph.D. Fellowship Program (for A. S.), by AGAUR via 2014 SGR 1554, by Fundació Cellex, and by CERCA Programme/Generalitat de Catalunya.

[1] M. Afzelius, N. Gisin, and H. de Riedmatten, Quantum Memory for Photons, Phys. Today 68, No. 12, 42 (2015).

[2] H. J. Kimble, The Quantum Internet, Nature (London) 453, 1023 (2008).

[3] A. G. Radnaev, Y. O. Dudin, R. Zhao, H. H. Jen, S. D. Jenkins, A. Kuzmich, and T. A. B. Kennedy, A Quantum Memory with Telecom-Wavelength Conversion, Nat. Phys. 6, 894 (2010).

[4] K. S. Choi, H. Deng, J. Laurat, and H. J. Kimble, Mapping Photonic Entanglement into and out of a Quantum Memory, Nature (London) 452, 67 (2008).

[5] S.-J. Yang, X.-J. Wang, X.-H. Bao, and J.-W. Pan, An Efficient Quantum Light-Matter Interface with Sub-Second Lifetime, Nat. Photonics 10, 381384 (2016).

[6] Y.-W. Cho, G. T. Campbell, J. L. Everett, J. Bernu, D. B. Higginbottom, M. T. Cao, J. Geng, N. P. Robins, P. K. Lam, and B.C. Buchler, Highly Efficient Optical Quantum Memory with Long Coherence Time in Cold Atoms, Optica 3, 100 (2016).

[7] B. Hensen, H. Bernien, A. E. Dreau, A. Reiserer, N. Kalb, M. S. Blok, J. Ruitenberg, R. F. L. Vermeulen, R. N. Schouten, C. Abellan et al., Loophole-Free Bell Inequality Violation Using Electron Spins Separated by 1.3 Kilometres, Nature (London) 526, 682 (2015).

[8] S. Yang et al., High-Fidelity Transfer and Storage of Photon States in a Single Nuclear Spin, Nat. Photonics 10, 507 (2016).

[9] D. G. England, K. A. G. Fisher, J.-P. W. MacLean, P. J. Bustard, R. Lausten, K. J. Resch, and B. J. Sussman, Storage and Retrieval of THz-Bandwidth Single Photons Using a Room-Temperature Diamond Quantum Memory, Phys. Rev. Lett. 114, 053602 (2015).

[10] R. Riedinger, S. Hong, R. A. Norte, J. A. Slater, J. Shang, A. G. Krause, V. Anant, M. Aspelmeyer, and S. Gröblacher, Non-classical Correlations between Single Photons and Phonons from a Mechanical Oscillator, Nature (London) 530, 313 (2016).

[11] M. P. Hedges, J. J. Longdell, Y. Li, and M. J. Sellars, Efficient Quantum Memory for Light, Nature (London) 465, 1052 (2010).

[12] D. Schraft, M. Hain, N. Lorenz, and T. Halfmann, Stopped Light at High Storage Efficiency in a $\mathrm{Pr}^{3+}: \mathrm{Y}_{2} \mathrm{SiO}_{5}$ Crystal, Phys. Rev. Lett. 116, 073602 (2016).

[13] G. Heinze, C. Hubrich, and T. Halfmann, Stopped Light and Image Storage by Electromagnetically Induced Transparency up to the Regime of One Minute, Phys. Rev. Lett. 111, 033601 (2013).

[14] M. Zhong, M. P. Hedges, R. L. Ahlefeldt, J. G. Bartholomew, S. E. Beavan, S. M. Wittig, J. J. Longdell, and M. J. Sellars, Optically Addressable Nuclear Spins in a Solid with a SixHour Coherence Time, Nature (London) 517, 177 (2015).

[15] E. Saglamyurek, N. Sinclair, J. Jin, J. A. Slater, D. Oblak, F. Bussières, M. George, R. Ricken, W. Sohler, and W. Tittel, 
Broadband Waveguide Quantum Memory for Entangled Photons, Nature (London) 469, 512 (2011).

[16] T. Zhong, J. M. Kindem, E. Miyazono, and A. Faraon, Nanophotonic Coherent Light-Matter Interfaces Based on Rare-Earth-Doped Crystals, Nat. Commun. 6, 8206 (2015).

[17] S. Marzban, J. G. Bartholomew, S. Madden, K. Vu, and M. J. Sellars, Observation of Photon Echoes from Evanescently Coupled Rare-Earth Ions in a Planar Waveguide, Phys. Rev. Lett. 115, 013601 (2015).

[18] G. Corrielli, A. Seri, M. Mazzera, R. Osellame, and H. de Riedmatten, Integrated Optical Memory Based on LaserWritten Waveguides, Phys. Rev. Applied 5, 054013 (2016).

[19] B. Lauritzen, J. Minar, H. de Riedmatten, M. Afzelius, N. Sangouard, C. Simon, and N. Gisin, TelecommunicationWavelength Solid-State Memory at the Single Photon Level, Phys. Rev. Lett. 104, 080502 (2010).

[20] E. Saglamyurek, J. Jin, V. B. Verma, M. D. Shaw, F. Marsili, S. W. Nam, D. Oblak, and W. Tittel, Quantum Storage of Entangled Telecom-Wavelength Photons in an ErbiumDoped Optical Fibre, Nat. Photonics 9, 83 (2015).

[21] K. De Greve et al., Quantum-Dot Spin-Photon Entanglement via Frequency Downconversion to Telecom Wavelength, Nature (London) 491, 421 (2012).

[22] B. Albrecht, P. Farrera, X. Fernandez-Gonzalvo, M. Cristiani, and H. de Riedmatten, A Waveguide Frequency Converter Connecting Rubidium-Based Quantum Memories to the Telecom C-Band, Nat. Commun. 5, 3376 (2014).

[23] N. Maring, K. Kutluer, J. Cohen, M. Cristiani, M. Mazzera, P. M. Ledingham, and H. de Riedmatten, Storage of UpConverted Telecom Photon in a Doped Crystal, New J. Phys. 16, 113021 (2014).

[24] P. Farrera, N. Maring, B. Albrecht, G. Heinze, and H. de Riedmatten, Nonclassical Correlations between a C-Band Telecom Photon and a Stored Spin-Wave, Optica 3, 1019 (2016).

[25] R. Ikuta, T. Kobayashi, K. Matsuki, S. Miki, T. Yamashita, H. Terai, T. Yamamoto, M. Koashi, T. Mukai, and N. Imoto, Heralded Single Excitation of Atomic Ensemble via SolidState-Based Telecom Photon Detection, Optica 3, 1279 (2016).

[26] C. Simon, H. de Riedmatten, M. Afzelius, N. Sangouard, H. Zbinden, and N. Gisin, Quantum Repeaters with Photon Pair Sources and Multimode Memories, Phys. Rev. Lett. 98, 190503 (2007).

[27] C. Clausen, I. Usmani, F. Bussières, N. Sangouard, M. Afzelius, H. de Riedmatten, and N. Gisin, Quantum Storage of Photonic Entanglement in a Crystal, Nature (London) 469, 508 (2011).

[28] D. Rieländer, K. Kutluer, P. M. Ledingham, M. Gündoğan, J. Fekete, M. Mazzera, and H. de Riedmatten, Quantum Storage of Heralded Single Photons in a PraseodymiumDoped Crystal, Phys. Rev. Lett. 112, 040504 (2014).

[29] W. Zhang, D.-S. Ding, S. Shi, Y. Li, Z.-Y. Zhou, B.-S. Shi, and G.-C. Guo, Storing a Single Photon as a Spin Wave Entangled with a Flying Photon in the Telecommunication Bandwidth, Phys. Rev. A 93, 022316 (2016).
[30] M. Afzelius, C. Simon, H. de Riedmatten, and N. Gisin, Multimode Quantum Memory Based on Atomic Frequency Combs, Phys. Rev. A 79, 052329 (2009).

[31] M. Gündoğan, P. M. Ledingham, K. Kutluer, M. Mazzera, and H. de Riedmatten, Solid State Spin-Wave Quantum Memory for Time-Bin Qubits, Phys. Rev. Lett. 114, 230501 (2015).

[32] P. Jobez, C. Laplane, N. Timoney, N. Gisin, A. Ferrier, P. Goldner, and M. Afzelius, Coherent Spin Control at the Quantum Level in an Ensemble-Based Optical Memory, Phys. Rev. Lett. 114, 230502 (2015).

[33] C. Laplane, P. Jobez, J. Etesse, N. Timoney, N. Gisin, and M. Afzelius, Multiplexed On-Demand Storage of Polarization Qubits in a Crystal, New J. Phys. 18, 013006 (2016).

[34] K. R. Ferguson, S. E. Beavan, J. J. Longdell, and M. J. Sellars, Generation of Light with Multimode Time-Delayed Entanglement Using Storage in a Solid-State Spin-Wave Quantum Memory, Phys. Rev. Lett. 117, 020501 (2016).

[35] J. Fekete, D. Rieländer, M. Cristiani, and H. de Riedmatten, Ultranarrow-Band Photon-Pair Source Compatible with Solid State Quantum Memories and Telecommunication Networks, Phys. Rev. Lett. 110, 220502 (2013).

[36] D. Rieländer, A. Lenhard, M. Mazzera, and H. de Riedmatten, Cavity Enhanced Telecom Heralded Single Photons for Spin-Wave Solid State Quantum Memories, New J. Phys. 18, 123013 (2016).

[37] P. Jobez, N. Timoney, C. Laplane, J. Etesse, A. Ferrier, P. Goldner, N. Gisin, and M. Afzelius, Towards Highly Multimode Optical Quantum Memory for Quantum Repeaters, Phys. Rev. A 93, 032327 (2016).

[38] See Supplemental Material at http://link.aps.org/ supplemental/10.1103/PhysRevX.7.021028 for details about the experimental setup, the preparation and characterization of the AFC, the storage sequences and the discussion of the results.

[39] H. Zhang, M. Sabooni, L. Rippe, C. Kim, S. Kröll, L. V. Wang, and P. R. Hemmer, Slow Light for Deep Tissue Imaging with Ultrasound Modulation, Appl. Phys. Lett. 100, 131102 (2012).

[40] S. E. Beavan, E. A. Goldschmidt, and M. J. Sellars, Demonstration of a Dynamic Bandpass Frequency Filter in a Rare-Earth Ion-Doped Crystal, J. Opt. Soc. Am. B 30, 1173 (2013).

[41] P. Sekatski, N. Sangouard, N. Gisin, H. de Riedmatten, and M. Afzelius, Photon-Pair Source with Controllable Delay Based on Shaped Inhomogeneous Broadening of Rare-Earth-Metal-Doped Solids, Phys. Rev. A 83, 053840 (2011).

[42] M. Sabooni, Q. Li, S. Kröll, and L. Rippe, Efficient Quantum Memory Using a Weakly Absorbing Sample, Phys. Rev. Lett. 110, 133604 (2013).

[43] P. Jobez, I. Usmani, N. Timoney, C. Laplane, N. Gisin, and M. Afzelius, Cavity-Enhanced Storage in an Optical Spin-Wave Memory, New J. Phys. 16, 083005 (2014). 